\title{
ON THE TOPOLOGY OF POSITIVELY CURVED KAEHLER MANIFOLDS II
}

\author{
R. L. BISHOP AND S. I. GOLDBËRG*
}

(Received August 2, 1965)

1. Introduction. A compact complex manifold of complex dimension $m$ possessing a Kaehler metric whose sectional curvatures are positive is analytically homeomorphic, for $m=1$ and 2 , with complex projective space $P C_{m}$. This is clear for $m=1$; for $m=2$, it has been shown by A. Andreotti and T. Frankel [6] by employing both differential and algebraic geometric methods. By restricting the metric further, M. Berger [1], recently obtained the following strong result:

Proposition 1. A complete Kaehler-Einstein manifold of positive curvature is isometric with $\left(P C_{m}, g_{0}\right)$ where $g_{0}$ is a metric of constant holomorphic curvature.

This statement is generalized below by an application of the following proposition due to the authors [4]:

PROPOSITION 2. The second betti number $b_{2}(M, R)$ of a complete Kaehler manifold $M$ of positive curvature (i.e., all of whose sectional curvatures in the Kaehlerian metric are positive) or of holomorphic pinching greater than $1 / 2$ is equal to 1.

We shall prove

THEOREM 1. A complete Kaehler manifold of positive curvature and constant scalar curvature is isometric with $\left(P C_{m}, g_{0}\right)$.

The sectional curvatures of a homogeneous Kaehlerian manifold of a compact Lie group with the invariant metric are all non-negative. If they are all positive, we have the

COROllaRY. A homogeneous Kaehlerian manifold of positive curvature is isometric with $\left(P C_{m}, g_{0}\right)$.

* This research was supported by the NSF under grant GP 3624 . 
An examination of the proof of Proposition 1 yields

THEOREM 2. A complete Kaehler manifold with holomorphic pinching greater than $1 / 2$ and constant scalar curvature is isometric with $\left(P C_{m}, g_{0}\right)$.

It is known that a $(2 m+1)$-dimensional sphere $S^{2 m+1}$ is a principal circle bundle over $P C_{m}$ with the circle $S^{1}$ as fibre. In $\$ 2$, a circle bundle $P$ over a complete Kaehler manifold of either positive curvature or holomorphic pinching greater than $1 / 2$ is constructed. Indeed, let $M$ be a compact Riemannian manifold of real dimension $n$ with metric $d s^{2}=\sum_{i=1}^{n}\left(\theta^{i}\right)^{2}$. Let $P$ be a circle bundle over $M$ with 1 -form $\gamma$ defining a connection in $P$. Moreover, let $a>0$ be a real number, and consider the Riemannian metric $d \sigma^{2}=\pi^{*} d s^{2}+a^{2} \gamma^{2}$ on $P$, where $\pi$ is the bundle projection $\pi: P \rightarrow M$. If $\left(P, d \sigma^{2}\right)$ is conformally flat, $P$ is conformally homeomorphic with a sphere. The exact homotopy sequences of the fibrings $S^{1} \rightarrow S^{2 m+1} \rightarrow P C_{m}$ and $S^{1} \rightarrow P \rightarrow M$ then show that $\pi_{i}(M)$ $\approx \pi_{i}\left(P C_{m}\right)$ for all $i$ when $M$ is simply connected. For Kaehlerian $M$ we have a much stronger result:

THEOREM 3. Let $\left(M, d s^{2}\right)$ be a complete Kaehler manifold of either positive curvature or holomorphic pinching greater than $1 / 2$. Then, if the principle circle bundle $\left(P, d \sigma^{2}\right)$ over $\left(M, d s^{2}\right)$ is conformally flat, $\left(M, d s^{2}\right)$ is isometric with $\left(P C_{m}, g_{0}\right)$.

Finally, by employing the above technique of passing to a circle bundle, we obtain (see [2, p. 256])

THEOREM 4. If $M$ is a complete Kaehler manifold of either positive curvature or holomorphic pinching greater than $1 / 2$, and $h$ is a positive lower bound on the mean curvature of $M$, then

$$
v(M) \leqq v\left(P C_{m}^{h}\right)
$$

where $v(M)$ is the volume of $M$ and $P C_{m}^{h}$ is complex projective $m$-space with a constant holomorphic curvature metric and mean curvature h. Equality is attained only if $M$ is isometric with $P C_{m}^{h}$.

2. Curvature relations. In this section we shall paraphrase some necessary facts, the details of which can be found in [8]. Let $P$ be a principal circle bundle over $M$ with projection $\pi$. Let $d s^{2}$ be a Riemannian metric on $M$ and $\gamma$ a 1 -form on $P$ defining a connection in $P$. Functions on $M$ will also 
be considered as functions on $P$ in a natural way, i.e., if $f$ is a function on $M$ we shall write $f$ rather than $\pi^{*} f$ when it is lifted to $P$. In the sequel, Latin indices $i, j, k, \cdots$ are to run from $1, \cdots, n$ and Greek indices $\alpha, \beta, \gamma, \cdots$ from $0,1, \cdots, n$.

Let $d \sigma^{2}=\pi^{*} d s^{2}+a^{2} \gamma^{2}$, where $a$ is a positive real number. Then $d \sigma^{2}$ is a Riemannian metric on $P$. Locally, i.e., on a small open set $U$, the metric $d s^{2}=\Sigma\left(\theta^{i}\right)^{2}$ where the $\theta^{i}, i=1, \cdots, n$ are 1 -forms defined on $U$. Let $\left(\omega_{j}^{i}\right)$ be a skew-symmetric matrix of 1 -forms on $U$ defining the Riemannian connection of $M$. Then the structure equations are

$$
d \theta^{i}=-\omega_{j}^{i} \wedge \theta^{j}
$$

and

$$
d \omega_{k}^{i}=-\omega_{j}^{i} \wedge \theta_{k}^{j}+\Omega_{k}^{i}
$$

where we sum on $j$ as in the usual convention. Set

$$
\Omega_{j}^{i}=\frac{1}{2} K_{i j k l} \theta^{k} \wedge \theta^{l}
$$

then the $K_{i j k l}$ are the components of the curvature tensor of $\left(M, d s^{2}\right)$ with respect to the basis $\theta^{i}$. Since the circle group is abelian, the structure equation of the connection defined by $\gamma$ is easily seen to be

$$
d \gamma=\pi^{*}\left(A_{i j} \theta^{i} \wedge \theta^{j}\right), \quad A_{i j}=-A_{j i}
$$

where the r.h.s. is the curvature form of the connection form $\gamma$. Clearly, the 2 -form $A_{i j} \theta^{i} \wedge \theta^{j}$ is closed.

If we put $\phi^{0}=a \gamma$ and $\phi^{i}=\pi^{*}\left(\theta^{i}\right)$, then

$$
d \sigma^{2}=\sum_{\alpha}\left(\phi^{\alpha}\right)^{2}
$$

LEMMA 1. If we set

$$
\begin{aligned}
& \psi_{0}^{0}=0, \\
& \psi_{0}^{i}=-\psi_{i}^{0}=-\sum_{j} a A_{i j} \phi^{j}, \\
& \psi_{j}^{i}=\pi^{*} \omega_{j}^{i}-A_{i j} \phi^{0},
\end{aligned}
$$

then the skew-symmetric matrix of 1-forms $\psi=\left(\psi_{\beta}^{\alpha}\right)$ defines the Riemannian cornection on $\left(P, d \sigma^{2}\right)$. 
LEMMA 2. If $\left(\Phi_{\beta}^{\alpha}\right)$ is the matrix of curvature forms of the connection defined by $\psi$, then

$$
\begin{aligned}
& \Phi_{0}^{0}=0, \\
& \Phi_{0}^{i}=-\Phi_{i}^{0}=-\sum_{k, l}\left\{a^{2} A_{i k} A_{k l} \varphi^{l} \wedge \varphi^{0}+a\left(D_{l} A_{i k}\right) \varphi^{l} \wedge \varphi^{k}\right\}, \\
& \Phi_{j}^{i}=\pi^{*} \Omega_{j}^{i}-a^{2}\left(A_{i j} A_{k l}+A_{i k} A_{j l}\right) \varphi^{k} \wedge \varphi^{l}-a\left(D_{k} A_{i j}\right) \phi^{k} \wedge \varphi^{0},
\end{aligned}
$$

where

$$
\left(D_{k} A_{i j}\right) \theta^{k}=d A_{i j}-A_{i k} \omega_{j}^{k}-A_{k j} \omega_{i}^{k}
$$

i.e., $D_{k} A_{i j}$ is the covariant derivative of the tensor field $A_{i j}$.

The proof follows directly from the structure equation

$$
\Phi_{\beta}^{\alpha}=d \psi_{\beta}^{\alpha}+\psi_{\gamma}^{\alpha} \wedge \psi_{\beta}^{\gamma}
$$

and lemma 1.

Set

$$
\Phi_{\beta}^{\alpha}=\frac{1}{2} \mathrm{R}_{\alpha \beta \gamma \delta} \varphi^{\gamma} \wedge \varphi^{\delta}
$$

then the $R_{\alpha \beta \gamma \delta}$ are the components of the curvature tensor of $\left(P, d \sigma^{2}\right)$ with respect to the basis $\varphi^{\alpha}$ of 1 -forms on $P$.

LEMMA 3. The curvature components of $\left(M, d s^{2}\right)$ and $\left(P, d \sigma^{2}\right)$ are related as follows:

$$
\begin{array}{ll}
\text { (a) } & R_{i j k l}=K_{i j k l}-a^{2}\left(2 A_{i j} A_{k l}+A_{i k} A_{j l}-A_{i l} A_{j k}\right), \\
\text { (b) } & R_{i o k o}=a^{2} \sum_{l} A_{i l} A_{k l}, \\
\text { (c) } & R_{i o k l}=-a D_{i} A_{k l} .
\end{array}
$$

Formulas (a) and (b) follow from lemma 2, and (c) is a consequence of lemma 2 together with the fact that $A_{i j} \theta^{i} \wedge \theta^{j}$ is closed.

3. Proofs of Theorems 1 and 2. Since curvature is positive or holomorphic pinching is greater than $1 / 2, b_{2}(M, R)=1$ by Proposition 2. Set $K_{i j}=\sum K_{i r j r}$ and let $J$ denote the almost complex structure tensor. Since the scalar 
curvature is constant, the Ricci 2 -form $\Phi$ of $\left(M, d s^{2}\right)$ is co-closed. For,

$$
\Phi=J_{j}^{r} K_{r i} \theta^{i} \wedge \theta^{j}
$$

and

$$
\delta \Phi=-i\left(\theta^{k}\right) D_{\mathbf{x}_{k}}\left(J_{j}^{\gamma} K_{r i} \theta^{i} \wedge \theta^{j}\right)=0
$$

since

$$
D_{s} K_{i}^{s}=\frac{1}{2} X_{i} K
$$

where $K=K_{i}^{i}$ is the scalar curvature and $\theta^{i}\left(X_{j}\right)=\delta_{j}^{i}$. But $\Phi$ is a closed form, and so it is harmonic. Thus, $\Phi$ is proportional to the fundamental form of $M$, i.e., $\left(M, d s^{2}\right)$ is an Einstein space. Applying Proposition 1, this gives Theorem 1.

Besides employing lemmas 1 (see [10, p. 170]) and 4 of [1], to obtain Proposition 1, the following statements, viz., Theorem 8.2 of [5] and the lemma of [4] are also used:

If the holomorphic sectional curvatures are non-negative, then a maximum curvature is holomorphic.

Let $\xi$ be a real form of bidegree $(1,1)$. Then, there exists an orthonormal basis $\left\{X_{i}, J X_{i}\right\}$ (where $J$ is the almost complex structure tensor) such that the only non-vanishing components of $\xi$ are of the form $\xi\left(X_{i}, J X_{i}\right)$.

The same technique gives Theorem 2. For, by reconstructing the proof of Proposition 1, it is seen that condition of positive curvature may be replaced by the condition that $M$ be holomorphically pinched with holomorphic pinching greater than $1 / 2$. Since $b_{2}(M, R)$ is still $1,\left(M, d s^{2}\right)$ is an Einstein space by the same reasoning as above. The Ricci curvature being positive, $M$ is compact by Myers' theorem and simply connected by a result of Kobayashi [9].

If $\left(M, d s^{2}\right)$ is homogeneous Kaehlerian with the invariant metric it is complete and has constant scalar curvature. Consequently, if all its sectional curvatures are positive, it is isometric with $\left(P C_{m}, g_{o}\right)$.

REMARK. Theorems 1 and 2 fail to hold if the condition on the scalar curvature is removed. This is clear for $m=1$. For $m>1$ one may construct a metric on complex projective space from the metric $g_{0}$ by merely adding $\epsilon d^{\prime} d^{\prime \prime} \rho$ to the fundamental form of $\left(P C_{m}, g_{0}\right)$, where $\rho$ is a real-analytic function on $P C_{m}$ which is not harmonic. By taking $\epsilon$ sufficiently small, the sectional curvatures of $P C_{m}$ remain positive, but in the new metric the scalar curvature is not constant. 
4. Proof of Theorem 3. In the sequel, $M$ is assumed to be a complete Kaehler manifold of either positive sectional curvature or holomorphic pinching greater than $1 / 2$. Let $\Omega$ be the fundamental 2 -form of $M$, and $Z$ be the ring of integers. By Proposition $2, H^{2}(M, R) \approx R$. The exact sequence $0 \rightarrow Z \rightarrow R$ $\rightarrow S^{1} \rightarrow 0$ induces an exact sequence of the cohomology groups of $M$ with coefficients in the corresponding sheaves of germs of sections. In particular, since $R$ is a fine sheaf

$$
H^{1}\left(M, \widetilde{S}^{1}\right) \approx H^{2}(M, Z)
$$

where $\widetilde{S}^{1}$ is the sheaf of germs of differentiable maps into $S^{1}$. As is wellknown, $H^{1}\left(M, \widetilde{S}^{1}\right)$ can be considered as the set of all principal circle bundles over $M$, and so since $H^{2}(M, Z) \approx Z, \Omega$ is a representative of a basis for $H^{2}(M, R)$. If the metric of $M$ is multiplied by a suitable scale factor, $\Omega$ represents the first Chern class of $M$. (This scale factor does not affect the result on volumes, since it also appears in the mean curvature and hence in the volume of the complex projective space to which we compare the volume of $M$.) Let $P$ be the circle bundle corresponding to this class, and $\gamma$ be a connection form such that $d \gamma=\pi^{*} \Omega$. If we put

$$
\Omega=J_{i j} \theta^{i} \wedge \theta^{j}
$$

and $A_{i j}=J_{i j}$ in lemma 3 , we obtain

PROPOSITION 3. Let $M$ be a complete Kaehler manifold whose sectional curvatures are all positive or which has holomorphic pinching greater than $1 / 2$. Then, there exists a principal circle bundle $P$ over $M$ and a Riemannian metric on $P$ whose curvature is related to that of $M$ as follows:

(a) $R_{i j k l}=K_{i j k l}-a^{2}\left(2 J_{i j} J_{k l}+J_{i k} J_{j l}-J_{i l} J_{j k}\right)$,

(b) $R_{\text {ioko }}=-R_{i o o k}=-R_{o i k o}=R_{o i o k}=a^{2} \delta_{i k}$,

(c) $R_{\alpha \beta \gamma \delta}=0$, otherwise.

LEMMA 4. Let $\bar{D}_{s}$ denote covariant differentiation with respect to the Riemannian connection on $P$. Then, relative to an orthonormal basis

(i) $\frac{1}{a} \bar{D}_{s} R_{i o k l}=K_{i r k l} J_{s}^{r}-a^{2}\left[\left(-2 \delta_{i s} J_{k l}+J_{i k} \delta_{s l}-J_{i l} \delta_{s k}\right)+\left(\delta_{i k} J_{l s}-\delta_{i l} J_{k s}\right)\right]$,

(ii) $\left(\bar{D}_{\rho} R_{i j k l}\right) \varphi^{\rho}=\left(D_{r} K_{i j k l}\right) \theta^{r}$.

Thus, if the l.h.s. of (i) vanishes, $M$ has constant holomorphic curvature. 
LEMMA 5 .

$$
\bar{D}_{s} R_{i o k l}=\bar{D}_{i} R_{s 0 k l} \text {. }
$$

This is an immediate consequence of lemma 4 .

LEMMA 6.

$$
\frac{1}{a} J_{r}^{s} \bar{D}_{s} R_{\alpha 0 \gamma \lambda}=R_{\alpha r \gamma \lambda}-a^{2}\left(\delta_{\alpha \gamma} \delta_{r \lambda}-\delta_{\alpha \lambda} \delta_{r \gamma}\right) .
$$

This follows from lemma 4 and Proposition 3.

Set $\quad R_{a \beta}=\sum_{\rho} R_{\alpha_{\rho} \beta \rho}$. Then, $R_{o i}=0$ and $R_{o o}=n a^{2}$. Thus, from lemma 6 ,

LEMMA 7.

$$
\frac{1}{a} J_{r}^{s} \bar{D}_{s} R_{o \gamma}=-R_{r \gamma}+n a^{2} \delta_{r \gamma}
$$

Note that $P$ is an Einstein space if its Ricci tensor is a parallel field or, more specifically, if $\bar{D}_{s} R_{o k}$ vanishes.

We now prove Theorem 3. Since $\left(P, d \sigma^{2}\right)$ is conformally flat, we have from [7, pp. 116 and 39]

$$
\bar{D}_{l} R_{o k}-\bar{D}_{k} R_{o l}=\bar{D}_{r} R_{o k l}^{r}=\frac{1}{n-1}\left(\bar{D}_{l} R_{o k}-\bar{D}_{k} R_{o l}\right)
$$

Since the r.h.s. of lemma 7 is symmetric, $\bar{D}_{l} R_{o k}$ is skew-symmetric. Thus,

$$
(n-1) \bar{D}_{l} R_{o k}=\bar{D}_{l} R_{o k}
$$

i.e., $\bar{D}_{l} R_{o k}=0$ for $n>2$, and so by the remark following lemma $7, P$ is an Einstein space. But a conformally flat Einstein space has constant curvature $a^{2}$, and so by Proposition $3(\mathrm{a}),\left(M, d s^{2}\right)$ has constant holomorphic curvature. Thus, since $M$ is compact and simply connected, $\left(M, d s^{2}\right)$ is isometric with $\left(P C_{m}, g_{o}\right)$. The case $n=2$ is trivial.

5. Proof of Theorem 4. We continue as above, with $P$ a principal circle bundle over $M$ and connection form $\gamma$ on $P$ such that $d \gamma=\pi^{*} \Omega$. Then, by Proposition 3, the components of the Ricci tensor of $P$ are 


$$
\begin{aligned}
& R_{i j}=K_{i j}-2 a^{2} \delta_{i j}, \\
& R_{i o}=0, \\
& R_{o o}=n a^{2} .
\end{aligned}
$$

Thus if $h$ is a lower bound for mean curvature on $M$, the smaller of $h-2 a^{2}$ and $n a^{2}$ is a lower bound for mean curvature on $P$. Let us choose $a^{2}$ so that this lower bound is as large as possible. Then $h-2 a^{2}=n a^{2}, a^{2}=h /(n+2)$, and $k=n h /(n+2)$ is a lower bound on the mean curvature of $P$.

It follows from [2, p. 256, Cor. 4] that the volume of $P$ is bounded above by the volume of the sphere $S^{n+1}(k / n)$ having constant curvature $k / n$, that is

$$
v(P) \leqq v\left(S^{n+1}(k / n)\right)
$$

Moreover, equality is attained only if $P$ has constant curvature, that is, if $P$ is isometric with $S^{n+1}(k / n)$. (The proof of this fact is obtained by computing bounds on the Jacobians of an exponential map of $P$, using the minimizing properties of Jacobi fields with respect to second variation, and integrating the bound obtained on the ball of radius equal to the diameter of $P$ in the tangent space, i.e., the domain of exp.)

Now, let us relate $v(M)$ and $v(P)$. Let $X_{o}$ be the vector field induced on $P$ by the action of $S^{1}$, so $\gamma\left(X_{0}\right)=1$ and $X_{0}$ is vertical. The integral curves of $X_{0}$ are periodic with period $2 \pi$, so the length of the fibres of $P$ in the metric $d \sigma^{2}$ is $2 \pi a$. Since $P$ is locally approximated by the Riemannian product $S^{1} \times U$, where $U$ is a small neighborhood of $M$, and $S^{1}$ is a circle of radius $a$, the volumes are related by

$$
2 \pi a v(M)=v(P) .
$$

We may apply this to the case $P=S^{n+1}(k / n)$, and $M=P C_{m}^{h}$ which has constant holomorphic curvature $H=2 h /(m+1)$. Then

$$
2 \pi a v\left(P C_{m}^{h}\right)=v\left(S^{n+1}(k / n)\right) .
$$

Inserting (2) and (3) in the inequality (1), the factors $2 \pi a$ cancel, leaving

$$
v(M) \leqq v\left(P C_{m}^{h}\right)
$$

Equality is attained only if $P$ is isometric with $S^{n+1}(k / n)$, and hence, by Proposition 3, only if $M$ is isometric with $P C_{m}^{h}$. 
REMARKS: (a) In the local complex coordinates $\left(z^{A}, \bar{z}^{A}\right)$,

$$
K_{A B^{*}} d z^{A} \wedge d \bar{z}^{B}=d^{\prime} d^{\prime \prime} \log G
$$

where $G=\operatorname{det} g\left(\frac{\partial}{\partial z^{A}}, \frac{\partial}{\partial \bar{z}^{B}}\right), g$ being the metric tensor of $M$. Therefore, replacing $d s^{2}$ by $c \cdot d s^{2},(c=$ const.), does not alter the Ricci curvature.

(b) The circle bundle $P$, obtained by reducing the canonical complex line bundle to $S^{1}$, over $M$ with positive mean curvature is simply connected [9], so there can be no improvement by passing to the simply connected covering of $P$.

\section{REFERENCES}

[1] M. BERGER, Sur quelques variétés riemanniennes compactes d'Einstein, Comptes rendus, 260 (1965), 1554-1557.

[2] R. L. BISHOP AND R. J CRITTENDEN, "Geometry of manifolds," Academic Press, New York, 1964.

[3] R. L. BISHOP AND S. I. GOLDBERG, On the topology of positively curved Kaehler manifolds, Tôhoku Math. Journ., 15(1963), 359-364.

[4] - On the second cohomology group of a Kaehler manifold of positive curvature, Proc. Amer. Math. Soc., 16(1965), 119-122.

[5] — Some implications of the generalized Gauss-Bonnet theorem, Trans. Amer. Math. Soc., 112(1964), 508-535.

[6] T. Frankel, Manifolds with positive curvature, Pacific Journ. Math., 11(1961), 165174.

[7] S. I. Goldberg, "Curvature and homology," Academic Press, New York, 1962.

[8] S. KoBAYASHI, Topology of positively pinched Kaehler manifolds, Tôhoku Math. Journ., 15(1963), 121-139.

[9] - On compact Kaehler manifolds with positive definite Ricci tensor, Ann. of Math., 74(1961), 570-574.

[10] K. Yano AND S. Bochner, "Curvature and Betti Numbers," Ann. of Math. Studies, No. 32, Princeton Univ. Press, Princeton, N. J., 1953.

UNIVERSITY OF ILLINOIS.

URBANA, ILLINOIS. 